\title{
The Impact of Preoperative Biliary Drainage on Postoperative Outcomes of Patients with Malignant Obstructive Jaundice: A Retrospective Analysis of 290 Consecutive Cases at a Single Medical Center
}

\author{
Gao Zhihui \\ Zhongshan Hospital Fudan University \\ Wang Jie \\ Zhongshan Hospital Fudan University \\ Shen Sheng \\ Zhongshan Hospital Fudan University \\ Bo Xiaobo \\ Zhongshan Hospital Fudan University \\ Suo Tao \\ Zhongshan Hospital Fudan University \\ Ni Xiaoling \\ Zhongshan Hospital Fudan University \\ Liu Han \\ Zhongshan Hospital Fudan University \\ Wang Yueqi \\ Zhongshan Hospital Fudan University \\ Huang Lihong \\ Zhongshan Hospital Fudan University \\ Houbao Liu ( $\nabla$ zsliuhb@sina.com ) \\ Zhongshan Hospital Fudan University
}

\section{Research Article}

Keywords: PBD, GBC, cholangiocarcinoma

Posted Date: April 27th, 2021

DOI: https://doi.org/10.21203/rs.3.rs-445511/v1

License: @ (i) This work is licensed under a Creative Commons Attribution 4.0 International License. Read Full License 


\section{Abstract}

Background

The efficacy of preoperative biliary drainage (PBD) has been debated for several decades, and indications for PBD are still a controversial topic. The aim of this study was to compare the efficacy of PBD among patients with malignant obstructive jaundice in current clinical settings.

Methods

All consecutive patients with malignant obstructive jaundice who underwent radical resection from June 2017 to December 2019 at Zhongshan Hospital were analyzed retrospectively. The study population was divided into two groups: a PBD group (PG) and direct surgery group (DG). Subgroups were chosen by site of obstruction.

Results

A total of 290 patients were analyzed. Postoperative complications occurred in 134 patients (overall rate: $46.4 \%$ ). Patients in group PG had a lower overall rate of postoperative complications as compared to group DG, with perioperative total bilirubin (TB) identified as an independent risk factor in multivariate analysis (hazard ratio $=1.004 ; 95 \%$ confidence interval $1.001-1.007 ; \mathrm{P}=0.017$ ). Subgroup analysis showed that PBD reduced the complication rate in patients with proximal obstruction. In the proximal-obstruction subgroup, a preoperative TB level $>162 \mu$ mol/L predicted postoperative complications.

\section{Conclusions}

PBD may reduce the overall rate of postoperative complications among patients with proximal obstructive jaundice.

Trial registration

ClinicalTrials.gov, 2018ZSLC 24. Registered May 17, 2018, https://clinicaltrials.gov/.

\section{Introduction}

Biliary obstruction often presents as the first symptom in several cancers affecting the region from the perihilar bile duct to the pancreatic head, including perihilar cholangiocarcinoma (pCCA), distal cholangiocarcinoma (dCCA), ampullary carcinoma, and pancreatic ductal adenocarcinoma (PDAC). Patients with intrahepatic cholangiocarcinoma (iCCA), gallbladder carcinoma (GBC), or other tumors invading the extrahepatic bile duct may also present with biliary obstruction as the first symptom [1-3]. However, these types of tumors are distinct in their presentation and natural history, as well as the approach to diagnosis and management[4]. Surgical treatment alone improves long-term survival of these patients, and the procedure is very challenging. Prolonged and progressive obstructive jaundice induces fatigue, malnutrition, bile stasis, cholangitis, and endotoxemia and is associated with hepatic dysfunction, coagulopathy, infections, anastomotic leakage, and delayed recovery after the surgical operation [5-7]. Preoperative biliary drainage (PBD) procedures have been introduced to alleviate the negative effects of biliary obstruction. Nonetheless, PBD is an invasive treatment and carries a risk of procedure-related complications. Although it has theoretical value, routine PBD does not improve perioperative outcomes in jaundiced patients. Indications for PBD in malignant obstructive jaundice are still debated $[8,9]$.

In China, many high-volume medical centers have achieved a significant reduction in surgical mortality and morbidity rates for radical resections, even for extended liver resection procedures and pancreatoduodenectomy in jaundiced patients. Preoperative management protocols of PBD may have played a substantial role in these improvements. Nevertheless, in which cases PBD should be recommended is still a matter of debate, and indications for PBD remain a controversial issue.

Therefore, the present study was aimed at assessing the impact of PBD on postoperative outcomes and at investigating the effectiveness of the currently recommended PBD protocol.

\section{Methods}

\section{Patients and study design}

Data on the patients that were consecutively referred for radical resection because of malignant biliary obstruction at Zhongshan Hospital Fudan University, Shanghai, China, from June 2017 to December 2019 were retrieved from the Hospital Information System and retrospectively analyzed. Only patients who achieved preoperative total bilirubin $>51 \mu \mathrm{mol} / \mathrm{L}$ were included. They either underwent a radical resection or received PBD before this surgical operation after admission. Only cases where PBD was carried out at our institution with full medical records were included. Demographic and clinical data were collected, including age, sex, the American Society of Anesthesiologists (ASA) score, body-mass index (BMI), medical history, histopathological diagnosis, preoperative physical examinations, laboratory tests, chest X-ray imaging, and computed tomography or magnetic resonance imaging of the abdomen. Informed written consent was obtained from each participating patient. A radical resection was considered complete if the entire gross tumor volume was removed with negative resection margins (R0 resection), whereas an incomplete resection was defined as the presence of a microscopic tumor in the surgical resection margin (R1 resection). Palliative resection was defined as the presence of any gross residual tumors (R2 resection), and such cases were excluded from the study. Finally, 290 patients were enrolled in the study, of whom 159 had PBD before the resection ("PBD group"; PG) and 131 had underwent an early surgical resection without PBD ("direct surgery group"; DG). The study protocol was approved by the ethics committees of Zhongshan Hospital and Fudan University. 
The methods were used in accordance with the approved guidelines. All postoperative complications were scored and classified using Common Terminology Criteria for Adverse Events 5.0.

\section{Results}

A total of 290 consecutive patients with malignant obstructive jaundice who underwent a radical resection at Zhongshan Hospital between June 2017 and December 2019 were included in the study. One hundred fifty-nine (54.8\%) patients received PBD (group PG) before the resection, and 131 (45.2\%) patients underwent a radical resection directly after admission (group DG). In group PG, for 5 (3.1\%), 5 (3.1\%), and 149 (93.7\%) out of 159 patients, EBS, ENBD, and PTBD were chosen as initial PBD, respectively. Eight patients (5.0\% of the 159) needed a second PBD, which was PTBD in all cases. The frequency of the second PBD was not significantly different among the 3 subgroups $(P=0.082$; Table 1$)$. Radical resections included pancreaticoduodenectomy, radical resection for $\mathrm{pCCA}$, and other procedures according to the tumor site.

\section{Patient characteristics}

Baseline characteristics of the patients with malignant obstructive jaundice in groups DG and PG are described in Table 2. The baseline characteristics (on admission) including age, sex, and BMI were not significantly different between the two groups. ASA scores were significantly different between the two groups: the PG group contained more patients with ASA score III-IV $(P<0.001)$. One hundred seventy-two $(59.1 \%)$ patients had proximal biliary obstruction, and $119(40.9 \%)$ distal biliary obstruction. The site of obstruction did not significantly affect the choice of PBD $(P=0.301)$. Final histopathological diagnoses were as follows: 4 iCCAs and 14 GBCs with invasion of an extrahepatic bile duct, 153 pCCAs, 51 distal biliary carcinomas, 47 ampullary carcinomas, 20 PDACs, and 1 hepatocellular carcinoma with invasion of an extrahepatic bile duct; prevalence rates of these diagnoses were not significantly different between groups DG and PG $(P=0.301)$.

\section{Laboratory testing results}

A comparison of these data between the time of initial diagnosis and the time point of perioperative testing is presented in Table 3. Within the DG group, initial laboratory test results including hemoglobin $(\mathrm{Hb})$ and ALB (albumin) were significantly higher as compared with the perioperative test $(P<0.001$ and $P=$ 0.016). ALT and AST levels also decreased significantly $(P<0.001$ and $P=0.005$, respectively). TB and direct bilirubin (DB) levels increased obviously (both $P$ $<0.001)$. PBD was found to prevent the deterioration of liver function. Within the PG group, Hb levels improved significantly $(P<0.001)$. ALT, AST, TB, and DB decreased significantly $(P<0.001, P<0.001, P<0.001$, and $P<0.001$, respectively).

A comparison of the laboratory test results between the two groups is presented in Table 4. At the time of initial diagnosis, the PG patients had severer anemia, higher percentage of neutrophils (NEUT \%), hypoalbuminemia, and jaundice $(P=0.015, P=0.001, P=0.038$, and $P<0.001$, respectively). As to perioperative laboratory findings, the PG patients' condition reversed in terms of neutrophils, hypoalbuminemia and jaundice $(P=0.050,<0.001$, and $<0.001$, respectively), but their anemia did not improve $(P<0.001)$.

\section{All postoperative outcomes and risk factors for postoperative complications}

The postoperative outcomes are shown in Table 5. The overall rate of postoperative complications was $46.4 \%$ (134 patients out of 290 ), and complications occurred more frequently in group DG $(P=0.029)$. The postoperative hemorrhage rate was significantly higher in group $D G(P=0.038)$, whereas postoperative delayed gastric emptying was significantly more frequent in group PG $(P=0.065)$. The rates of mortality and of other complications were similar between the two groups. The location of obstruction was used to decide on the main routine surgical procedure, and each group was divided into a proximal-obstruction subgroup and distal-obstruction subgroup. The overall rate of postoperative complications in the combined proximal-obstruction group was $77.9 \%$ (Table 6), and complications occurred more frequently in the proximal-obstruction DG subgroup $(P=0.038$; Table 7$)$. In the combined distal-obstruction group, the overall rate of postoperative complications was $50.8 \%$ (Table 6), and PBD did not affect this rate $(P=0.249$, Table 7).

In univariate analysis, PBD proved to be associated with better postoperative outcomes $(P=0.025$; Table 8$)$. Perioperative Hb and perioperative TB were associated with postoperative complications $(P=0.027$ and $P=0.016$, respectively). Multivariate analysis indicated that perioperative TB is an independent risk factor for postoperative complications (Table 8).

Preoperative TB concentration greater than $162 \mu \mathrm{mol} / \mathrm{L}$ distinguished patients with postoperative complication(s) from those without (Fig. 1, ROC curve analysis). For this cutoff, the area under the ROC curve was 0.7024 ( $95 \%$ confidence interval $0.588-0.816)(P=0.002$, sensitivity $62.8 \%$, specificity $74.4 \%$ ).

\section{Discussion}

In this retrospective study, we analyzed the efficacy of PBD in patients with malignant obstructive jaundice in current clinical settings. It sounded confusing to see patients with a number of different malignancies. However, we want to emphasize that patients often do not have full medical examination at their first visit but require receiving preliminary treatment with limited data. So we want to find some easily accessible indicators to help patients receive treatments as early as possible.

Obstructive jaundice is a life-threatening problem in patients with malignant tumors. Although the degree of hyperbilirubinemia is not necessarily related to a tumor stage, hyperbilirubinemia interferes with many organ functions and limits treatment choices. Bilirubin, as a product of heme metabolism, is nearly nontoxic in its normal physiological range of concentrations, whereas hyperbilirubinemia is toxic and should be taken seriously. In particular, it has been associated with an increased risk of postoperative complications. PBD is a kind of "bridge therapy" before a radical surgical resection is attempted for malignant obstructive jaundice. There is no consensus on indications for PBD so far.

Page $3 / 9$ 
The retrospective data showed that PBD may reduce the overall rate of postoperative complications among patients with proximal obstructive jaundice. Perioperative TB turned out to be an independent risk factor for postoperative complications. Preoperative TB greater than $162 \mu \mathrm{mol} / \mathrm{L}$ distinguished patients with postoperative complications from those without.

PBD has had varied effects in different studies. To be exact, different PBD options have manifested various effects in different cancers. It must be emphasized that the data published so far came from diverse studies, where the patients had a small number of cancer types affecting the biliary tree. In 2010, van der Gaag N.A. et al. conducted a multicenter randomized controlled trial regarding the cancer of the pancreatic head and demonstrated that routine PBD increases the rate of complications. This conclusion was based on the bilirubin range 40 to $250 \mu \mathrm{mol} / \mathrm{L}$ and was made about the cancer of the pancreatic head [10]. In 2016, a meta-analysis suggested that the PBD group has significantly fewer major adverse effects than the direct surgery group. This conclusion was made about a series of tumors, e.g., cancers of the biliary tract, cancers of the head and neck, and cancers of the duodenum. On the other hand, the studies included in this meta-analysis took place between 1981 and 2011. Nearly at the same time, another meta-analysis suggested that PBD worsens postoperative outcomes, increasing the prevalence rate of infectious complications, surgical site infections, and delayed gastric emptying [11]. Few studies have been conducted on multicenter randomized controlled trials for malignant obstructive jaundice. Actually, in recent years, several PBD methods were simultaneously employed, and new drainage brackets are coming onto the scene [12,13]. The management of obstructive jaundice is complicated and involves not only surgeons and medical centers but also the entire healthcare system [14]. Hegel's principle "what exists is reasonable" is thought to be applicable worldwide, but we found a more accurate observation by examining the current methods in some clinical settings. For example, a Japanese retrospective study covered 10-year clinical experience and revealed that PBD reduces postoperative morbidity and mortality in the whole country regardless of the PBD type [15]. This finding makes us think that we can also find a beneficial modality at our medical center. In the 2 years under study, we applied a mature surgical technology and advanced equipment, and the observed prevalence of postoperative complications is not consistent with other hospitals and countries.

Our results show a clear difference made by PBD among patients with malignant obstructive jaundice. This report is the first retrospective study at the latest level of technology to show benefits of PBD for patients with malignant obstructive jaundice in China. Accurate preoperative diagnosis of biliary tract tumors is difficult, but the estimation of an obstruction site is easier. On the basis of our study, we believe that patients with proximal obstruction should consider PBD more often. Due to the limited number of cases in the 2 recent years under study, we did not obtain strong evidence that PBD affects postoperative complications of patients with distal obstruction.

Concerning limitations of this study, the major problem with retrospective analysis is biased selection of patients. At the time of initial diagnosis, the PG group patients had severer anemia, infection, hypoalbuminemia, and jaundice. Although the best way to reduce or eliminate the selection bias is a randomized controlled trial, such a trial is not feasible for patients with malignant obstructive jaundice. Only retrospective analysis can help physicians choose the best option for these patients.

\section{Conclusion}

Our study indicates that PBD may reduce the overall rate of postoperative complications in patients with proximal obstructive jaundice. Perioperative TB is an independent risk factor for postoperative complications and supports the decision to recommend preoperative PBD when preoperative TB is $>162 \mu \mathrm{mol} / \mathrm{L}$.

\section{Declarations}

Authors' contributions

Houbao Liu and Sheng Shen conceived the study. Zhihui Gao and Huang Lihong analyzed and interpreted the data. Zhihui Gao performed the writing of the manuscript. Wang Jie, Bo Xiaobo, Suo Tao, Ni Xiaoling, Liu Han and Wang Yueqi collected and assembled the data. Houbao Liu submitted the manuscript and is the corresponding author. All authors read and approved the final manuscript.

Funding

This study was supported by Clinical research in Zhongshan Hospital, No. 2018ZSLC24.

Availability of data and materials

The data used to support the findings of this study are available from the corresponding author upon request.

Ethics approval and consent to participate

This study was approved by the ethics committees of Zhongshan Hospital and Fudan University. All patients signed informed consent.

Consent for publication

The patient was given his consent for information about himself to be published in World Journal of Surgical Oncology.

Competing interests

The authors declare that they have no competing interests. 


\section{References}

1. Krasinskas AM. Cholangiocarcinoma. Surg Pathol Clin. 2018 2018-06-01;11(2):403 - 29.

2. Oliveira IS, Kilcoyne A, Everett JM, Mino-Kenudson M, Harisinghani MG, Ganesan K. Cholangiocarcinoma: classification, diagnosis, staging, imaging features, and management. Abdom Radiol (NY). 2017 2017-06-01;42(6):1637-49.

3. Lee AJ, Chun YS. Intrahepatic cholangiocarcinoma: the AJCC/UICC 8th edition updates. Chin Clin Oncol. 2018 2018-10-01;7(5):52.

4. Waseem D, Tushar P. Intrahepatic, perihilar and distal cholangiocarcinoma: Management and outcomes. ANN HEPATOL. 2017 2017-01-20;16(1):133-9.

5. Kendall T, Verheij J, Gaudio E, et al. Anatomical, histomorphological and molecular classification of cholangiocarcinoma. LIVER INT. 2019 2019-05-01;39 Suppl 1:7-18.

6. Lee RM, Maithel SK. Approaches and Outcomes to Distal Cholangiocarcinoma. SURG ONCOL CLIN N AM. 2019 2019-10-01;28(4):631-43.

7. Waseem D, Tushar P. Intrahepatic, perihilar and distal cholangiocarcinoma: Management and outcomes. ANN HEPATOL. 2017 2017-01-20;16(1):133-.

8. Moole H, Bechtold M, Puli SR. Efficacy of preoperative biliary drainage in malignant obstructive jaundice: a meta-analysis and systematic review. WORLD J SURG ONCOL. 2016;14(1):182. 2016-07-11.

9. Neuhaus H. Preoperative biliary drainage in hilar cholangiocarcinoma: when and how? Endosc Int Open. 2020 2020-02-01;8(2):E211-3.

10. van der Gaag NA, Rauws E, A,van Eijck CH, Bruno MJ, van der Harst E, Kubben FJ, Gerritsen JJ, et al. J Preoperative biliary drainage for cancer of the head of the pancreas. N Engl J Med. 2010 2010-01-14;362(2):129-37.

11. Celotti A, Solaini L, Montori G, Coccolini F, Tognali D, Baiocchi G. Preoperative biliary drainage in hilar cholangiocarcinoma: Systematic review and metaanalysis Eur J Surg Oncol 43 1628-35.

12. Liu JG, Wu J, Wang J, Shu GM, Wang YJ, Lou C, Zhang J, Du Z. Endoscopic Biliary Drainage Versus Percutaneous Transhepatic Biliary Drainage in Patients with Resectable Hilar Cholangiocarcinoma: A Systematic Review and Meta-Analysis J Laparoendosc Adv Surg Tech A $281053-60$.

13. Tringali A, Boskoski I, Costamagna G. Endoscopic Stenting in Hilar Cholangiocarcinoma: When, How, and How Much to Drain? Gastroenterol Res Pract 20195161350.

14. Shin SH, Han IW, Ryu Y, Kim N, Choi DW, Heo JS. Optimal timing of pancreaticoduodenectomy following preoperative biliary drainage considering major morbidity and postoperative survival J Hepatobiliary Pancreat Sci 26 449-58.

15. Chaudhary RJ, Higuchi R, Nagino M, Unno M, Ohtsuka M, Endo I, Hirano S, et al. Survey of preoperative management protocol for perihilar cholangiocarcinoma at 10 Japanese high-volume centers with a combined experience of 2,778 cases J Hepatobiliary Pancreat Sci $26490-502$.

\section{Tables}

Table 1. The frequency of a second PBD in group PG

\begin{tabular}{|lllllll|}
\hline & Total & EBS & ENBD & PTBD & Statistic & $P$ value \\
\cline { 1 - 5 } First PBD & $159(100 \%)$ & $5(3.1 \%)$ & $5(3.1 \%)$ & $149(93.7 \%)$ & \multirow{2}{*}{$\chi^{2}=5.665$} & $0.082^{*}$ \\
\cline { 1 - 5 } Second PBD & $8(5.0 \%)$ & $1(20.0 \%)$ & $1(20.0 \%)$ & $6(4.0 \%)$ & & \\
\hline
\end{tabular}

*Fisher's exact test in Monte Carlo mode.

Table 2. Demographic data on the patients under study 


\begin{tabular}{|c|c|c|c|c|c|c|}
\hline \multicolumn{2}{|l|}{ Characteristics } & $\begin{array}{l}\text { Total } \\
(n=290)\end{array}$ & \multirow{2}{*}{$\begin{array}{l}\text { DG } \\
(n=131)\end{array}$} & \multirow{2}{*}{$\begin{array}{l}P G \\
(n=159)\end{array}$} & statistic & \multirow[t]{2}{*}{$P$ value } \\
\hline \multirow{5}{*}{ Age (years) } & & & & & \multirow{3}{*}{$t=0.007$} & \\
\hline & Mean $\pm S D$ & $62.21 \pm 9.41$ & $62.21 \pm 9.60$ & $62.2 \pm 9.30$ & & \multirow[t]{2}{*}{0.994} \\
\hline & (range) & $(24-80)$ & $(24-80)$ & $(28-80)$ & & \\
\hline & $\geq 70, n(\%)$ & $63(21.72 \%)$ & $29(22.14 \%)$ & $34(21.38 \%)$ & \multirow{2}{*}{$\begin{array}{l}x^{2}= \\
0.024\end{array}$} & \multirow[t]{2}{*}{0.877} \\
\hline & $<70, \mathrm{n}(\%)$ & $227(78.28 \%)$ & $102(77.86 \%)$ & $125(78.62 \%)$ & & \\
\hline \multirow[t]{2}{*}{ Sex } & Male, n (\%) & $185(63.79 \%)$ & $82(62.60 \%)$ & $103(64.78 \%)$ & \multirow{2}{*}{$\begin{array}{l}x^{2}= \\
0.148\end{array}$} & \multirow[t]{2}{*}{0.700} \\
\hline & Female, n (\%) & $105(36.21 \%)$ & $49(37.40 \%)$ & $56(35.22 \%)$ & & \\
\hline BMI $\left(\mathrm{kg} / \mathrm{m}^{2}\right)$ & Mean $\pm S D$ & $22.72 \pm 3.02$ & $22.52 \pm 2.65$ & $22.86 \pm 3.25$ & $t=0.862$ & 0.389 \\
\hline \multirow[t]{3}{*}{ ASA score } & I & $22(7.59 \%)$ & $10(7.63 \%)$ & $12(7.55 \%)$ & \multirow{3}{*}{$\begin{array}{l}x^{2}= \\
17.810\end{array}$} & \multirow[t]{3}{*}{$<0.001$} \\
\hline & ॥ & $237(81.72 \%)$ & $118(90.08 \%)$ & $119(74.84 \%)$ & & \\
\hline & III-IV & 31 (10.69\%) & $3(2.29 \%)$ & $28(17.61 \%)$ & & \\
\hline \multirow[t]{4}{*}{ Medical history } & hepatolithiasis & 12 & 2 & 10 & \multirow{4}{*}{$\begin{array}{l}x^{2}= \\
1.752\end{array}$} & \multirow[t]{4}{*}{$0.636^{*}$} \\
\hline & cholecystolithiasis & 29 & 11 & 18 & & \\
\hline & HBP & 50 & 17 & 33 & & \\
\hline & Diabetes & 26 & 8 & 18 & & \\
\hline \multirow{2}{*}{$\begin{array}{l}\text { Obstruc- } \\
\text { Tion site }\end{array}$} & Proximal & 172 & 82 & 90 & \multirow{2}{*}{$\begin{array}{l}x^{2}= \\
1.068\end{array}$} & \multirow[t]{2}{*}{0.301} \\
\hline & Distal & 118 & 49 & 69 & & \\
\hline \multirow{7}{*}{$\begin{array}{l}\text { Histopa- } \\
\text { thological diagnosis }\end{array}$} & iCCA & 4 & 3 & 1 & \multirow{7}{*}{$\begin{array}{l}x^{2}= \\
4.187\end{array}$} & \multirow[t]{7}{*}{$0.664^{*}$} \\
\hline & GBC & 14 & 8 & 6 & & \\
\hline & $\mathrm{pCCA}$ & 153 & 71 & 82 & & \\
\hline & biliary carcinoma & 51 & 21 & 30 & & \\
\hline & ampullary carcinoma & 47 & 21 & 26 & & \\
\hline & PDAC & 20 & 7 & 13 & & \\
\hline & hepatocellular carcinoma & 1 & 0 & 1 & & \\
\hline
\end{tabular}

*Fisher's exact test in Monte Carlo mode.

HBP: high blood pressure.

Table 3. A comparison of laboratory testing results between two time points (initial diagnosis and perioperative testing) within each group 


\begin{tabular}{|c|c|c|c|c|c|c|c|c|c|c|}
\hline \multirow[t]{2}{*}{ Parameters } & \multicolumn{2}{|l|}{ DG } & \multicolumn{3}{|l|}{ Within DG } & \multicolumn{2}{|l|}{ PG } & \multicolumn{3}{|l|}{ Within PG } \\
\hline & initial & perioperative & $\begin{array}{l}\text { cases } \\
\text { with } \\
\text { paired } \\
\text { data } \\
\text { available }\end{array}$ & statistic & $\begin{array}{l}P \\
\text { value }\end{array}$ & initial & perioperative & $\begin{array}{l}\text { cases } \\
\text { with } \\
\text { paired } \\
\text { data } \\
\text { available }\end{array}$ & statistic & $\begin{array}{l}P \\
\text { value }\end{array}$ \\
\hline $\mathrm{Hb}(\mathrm{g} / \mathrm{L})$ & $126.13 \pm 14.34$ & $122.97 \pm 14.71$ & 124 & $t=5.400$ & $<0.001$ & $121.47 \pm 17.51$ & $114.70 \pm 15.78$ & 147 & $t=6.352$ & $<0.001$ \\
\hline $\begin{array}{l}\text { WBC } \\
\left(10^{\wedge} 9 / L\right)\end{array}$ & $6.12 \pm 1.84$ & $5.98 \pm 1.75$ & 124 & $t=1.463$ & 0.146 & $6.45 \pm 2.10$ & $6.34 \pm 2.00$ & 147 & $t=0.639$ & 0.524 \\
\hline NEUT (\%) & $63.10 \pm 9.36$ & $64.23 \pm 9.31$ & 124 & $t=-1.789$ & 0.076 & $66.94 \pm 9.27$ & $62.30 \pm 9.37$ & 146 & $t=4,705$ & 0.028 \\
\hline $\begin{array}{l}\text { Plt } \\
\left(10^{\wedge} 9 / \mathrm{L}\right)\end{array}$ & $252.23 \pm 83.36$ & $254.86 \pm 85.68$ & 124 & $t=-0.751$ & 0.454 & $253.24 \pm 87.03$ & $243.80 \pm 83.14$ & 147 & $t=1.764$ & $<0.001$ \\
\hline ALB (g/L) & $38.82 \pm 4.09$ & $38.17 \pm 4.05$ & 121 & $t=2.435$ & 0.016 & $37.43 \pm 4.99$ & $40.70 \pm 5.22$ & 147 & $t=-5.914$ & 0.100 \\
\hline $\operatorname{ALT}(\mathrm{U} / \mathrm{L})$ & $200.21 \pm 151.89$ & $165.80 \pm 140.32$ & 122 & $t=4.517$ & $<0.001$ & $166.09 \pm 152.17$ & $78.52 \pm 59.40$ & 147 & $t=7.656$ & $<0.001$ \\
\hline AST (U/L) & $133.94 \pm 99.47$ & $115.38 \pm 93.63$ & 122 & $t=2.887$ & 0.005 & $131.58 \pm 122.80$ & $54.60 \pm 43.18$ & 146 & $t=7.882$ & 0.001 \\
\hline $\begin{array}{l}\text { TB } \\
(\mu \mathrm{mol} / \mathrm{L})\end{array}$ & $140.99 \pm 83.27$ & $158.12 \pm 95.11$ & 122 & $t=-4.572$ & $<0.001$ & $227.38 \pm 126.49$ & $83.03 \pm 57.59$ & 147 & $t=14.399$ & $<0.001$ \\
\hline $\begin{array}{l}\mathrm{DB} \\
(\mu \mathrm{mol} / \mathrm{L})\end{array}$ & $116.11 \pm 70.21$ & $133.40 \pm 79.21$ & 121 & $t=-5.402$ & $<0.001$ & $186.06 \pm 103.03$ & $71.49 \pm 49.17$ & 147 & $t=13.904$ & $<0.001$ \\
\hline GGT (U/L) & $798.17 \pm 602.69$ & $739.93 \pm 593.06$ & 119 & $t=3.228$ & 0.002 & $586.88 \pm 436.73$ & $228.60 \pm 181.69$ & 145 & $\mathrm{t}=11.031$ & $<0.001$ \\
\hline $\operatorname{ALP}(\mathrm{U} / \mathrm{L})$ & $434.24 \pm 268.60$ & $423.75 \pm 262.36$ & 122 & $t=1.031$ & 0.305 & $465.28 \pm 363.83$ & $230.40 \pm 158.91$ & 145 & $t=9.484$ & $<0.001$ \\
\hline $\begin{array}{l}\mathrm{Na} \\
(\mathrm{mmol} / \mathrm{L})\end{array}$ & $139.83 \pm 3.01$ & $139.74 \pm 3.16$ & 119 & $t=0.460$ & 0.646 & $138.87 \pm 3.16$ & $139.78 \pm 2.96$ & 146 & $t=-3.279$ & $<0.001$ \\
\hline PT (s) & $11.35 \pm 1.42$ & $11.20 \pm 1.22$ & 117 & $t=0.843$ & 0.401 & $11.59 \pm 1.51$ & $11.65 \pm 1.08$ & 146 & $t=-0.402$ & 0.682 \\
\hline
\end{tabular}

Hb: hemoglobin; WBC: white blood cell; NEUT: neutrophils; PIt: platelet; ALB: albumin; ALT: alanine transminase-glutamic pyruvic transaminase; AST: aspartate transminase-glutamic oxalo-acetic transaminase; TB: total bilirubin; DB: direct bilirubin; GGT: y-glutamyltransferase; ALP: alkaline phosphatase; Na; natrium; PT: prothrombin time

Table 4. A comparison of laboratory testing results at two time points (initial diagnosis and perioperative testing) between the two groups

\begin{tabular}{|c|c|c|c|c|c|c|c|c|}
\hline \multirow[t]{2}{*}{ Characteristics } & \multicolumn{2}{|l|}{ DG } & \multicolumn{2}{|l|}{ PG } & \multicolumn{2}{|c|}{$\begin{array}{l}\text { Initial data compared } \\
\text { between groups }\end{array}$} & \multicolumn{2}{|c|}{$\begin{array}{l}\text { Perioperative data compared } \\
\text { between groups }\end{array}$} \\
\hline & initial & perioperative & initial & perioperative & statistic & $P$ value & statistic & $P$ value \\
\hline $\mathrm{Hb}(\mathrm{g} / \mathrm{L})$ & $125.85 \pm 14.41$ & $123.11 \pm 14.74$ & $121.17 \pm 17.58$ & $115.35 \pm 15.93$ & $t=2.441$ & 0.015 & $t=4.193$ & $<0.001$ \\
\hline WBC $\left(10^{9} / \mathrm{L}\right)$ & $6.11 \pm 1.82$ & $6.00 \pm 1.76$ & $6.55 \pm 2.26$ & $6.37 \pm 2.04$ & $t=-1.754$ & 0.081 & $t=-1.670$ & 0.096 \\
\hline NEUT (\%) & $63.15 \pm 9.44$ & $64.16 \pm 9.31$ & $67.11 \pm 9.40$ & $61.9 \pm 9.51$ & $t=-3.504$ & 0.001 & $t=1.972$ & 0.050 \\
\hline Plt $\left(10^{9} / \mathrm{L}\right)$ & $250.5 \pm 82.90$ & $255.24 \pm 85.44$ & $255.6 \pm 88.94$ & $248.31 \pm 84.76$ & $t=-0.494$ & 0.662 & $t=0.679$ & 0.498 \\
\hline $\operatorname{ALB}(\mathrm{g} / \mathrm{L})$ & $38.57 \pm 4.16$ & $38.21 \pm 4.06$ & $37.42 \pm 4.96$ & $40.75 \pm 5.23$ & $t=2.085$ & 0.038 & $t=-4.552$ & $<0.001$ \\
\hline $\operatorname{ALT}(\mathrm{U} / \mathrm{L})$ & $198.78 \pm 153.36$ & $165.80 \pm 140.32$ & $166.10 \pm 150.58$ & $79.19 \pm 62.58$ & $t=1.799$ & 0.073 & $t=6.340$ & $<0.001$ \\
\hline AST (U/L) & $134.50 \pm 99.18$ & $115.38 \pm 93.63$ & $131.63 \pm 121.91$ & $55.24 \pm 44.08$ & $t=0.140$ & 0.889 & $t=6.530$ & $<0.001$ \\
\hline $\mathrm{TB}(\mu \mathrm{mol} / \mathrm{L})$ & $140.84 \pm 82.94$ & $158.12 \pm 95.11$ & $225.1 \pm 126.27$ & $81.76 \pm 56.74$ & $t=-6.695$ & $<0.001$ & $t=7.837$ & $<0.001$ \\
\hline $\mathrm{DB}(\mu \mathrm{mol} / \mathrm{L})$ & $115.70 \pm 69.55$ & $132.52 \pm 79.48$ & $184.20 \pm 102.96$ & $70.36 \pm 48.47$ & $t=-6.601$ & $<0.001$ & $t=7.598$ & $<0.001$ \\
\hline GGT (U/L) & $812.81 \pm 627.21$ & $736.18 \pm 592.00$ & $582.53 \pm 434.59$ & $224.00 \pm 178.62$ & $t=3.501$ & 0.001 & $t=9.160$ & $<0.001$ \\
\hline $\operatorname{ALP}(\mathrm{U} / \mathrm{L})$ & $430.06 \pm 265.00$ & $423.75 \pm 262.36$ & $465.36 \pm 362.82$ & $230.09 \pm 157.57$ & $t=-0.936$ & 0.350 & $t=7.195$ & $<0.001$ \\
\hline $\mathrm{Na}(\mathrm{mmol} / \mathrm{L})$ & $139.74 \pm 3.03$ & $139.75 \pm 3.15$ & $138.88 \pm 3.18$ & $139.87 \pm 2.96$ & $t=2.303$ & 0.022 & $t=-0.327$ & 0.728 \\
\hline PT (s) & $11.53 \pm 2.12$ & $11.16 \pm 1.21$ & $11.569 \pm 1.50$ & $11.69 \pm 1.09$ & $t=-0.171$ & 0.864 & $t=-3.824$ & $<0.001$ \\
\hline
\end{tabular}

Table 5. All postoperative complications in the study population 


\begin{tabular}{|llllll|}
\hline & $\begin{array}{l}\text { Total } \\
(\mathrm{n}=290)\end{array}$ & $\begin{array}{l}\text { DG } \\
(\mathrm{n}=131)\end{array}$ & $\begin{array}{l}\text { PG } \\
(\mathrm{n}=159)\end{array}$ & statistic & Pvalue \\
\hline All complications & 134 & 70 & 64 & $\chi^{2}=5.002$ & 0.025 \\
\hline Intestinal fistula & 23 & 10 & 13 & $\chi^{2}=0.029$ & 0.865 \\
\hline Pancreatic fistula & 20 & 10 & 10 & $\chi^{2}=0.202$ & 0.653 \\
\hline Biliary fistula & 1 & 0 & 1 & - & - \\
\hline Gastrointestinal anastomotic fistula & 2 & 0 & 2 & - & - \\
\hline Abdominal liquid accumulation & 31 & 16 & 15 & $\chi^{2}=0.581$ & 0.446 \\
\hline Delayed gastric emptying & 17 & 4 & 13 & $\chi^{2}=3.415$ & 0.065 \\
\hline Hemorrhage & 21 & 14 & 7 & $\chi^{2}=4.223$ & 0.040 \\
\hline Hepatic failure & 6 & 3 & 3 & - & 1.000 \\
\hline Reoperation & 11 & 6 & 5 & $\chi^{2}=0.406$ & 0.552 \\
\hline Incision complication & 8 & 3 & 5 & $\chi^{2}=0.196$ & 0.733 \\
\hline Mortality & 6 & 3 & 3 & - & 1.000 \\
\hline Sepsis & 78 & 41 & 37 & $\chi^{2}=2.354$ & 0.125 \\
\hline
\end{tabular}

Table 6. Postoperative outcomes in proximal- and distal-obstruction groups

\begin{tabular}{|lllll|}
\hline & Proximal obstruction & Distal obstruction & statistic & $P$ value \\
\hline Total & 172 & 118 & $\chi^{2}=23.141$ & $<0.001$ \\
All complications & 134 & 60 & & \\
$(\%)$ & $(77.9 \%)$ & $(50.8 \%)$ & & \\
\hline
\end{tabular}

Table 7. Postoperative outcomes by subgroups

\begin{tabular}{|c|c|c|c|c|c|}
\hline & Total & DG & PG & statistic & $P$ value \\
\hline Proximal obstruction (number of patients) & 172 & 82 & 90 & \multirow[t]{2}{*}{$x^{2}=4.295$} & \multirow[t]{2}{*}{0.038} \\
\hline Complication(s) (number of patients) & 134 & 70 & 64 & & \\
\hline Distal obstruction & 118 & 49 & 69 & \multirow{2}{*}{$x^{2}=1.329$} & \multirow[t]{2}{*}{0.249} \\
\hline Complication(s) & 60 & 28 & 32 & & \\
\hline
\end{tabular}

Table 8. Univariate and multivariate analyses of factors possibly associated with postoperative outcomes in the study population (290 patients) 


\begin{tabular}{|lllllll|}
\hline & \multicolumn{3}{l}{ Univariate analysis } & \multicolumn{3}{l|}{ Multivariate analysis } \\
\hline & HR & $95 \% \mathrm{Cl}$ & P value & HR & $95 \% \mathrm{Cl}$ & P value \\
\hline Age>70 & 0.831 & $0.456-1.515$ & 0.545 & & & \\
\hline Gender & 0.971 & $0.601-1.570$ & 0.906 & & \\
\hline Tumor site & 0.970 & $0.606-1.553$ & 0.900 & & 0.163 \\
\hline PBD & 0.587 & $0.368-0.937$ & 0.025 & & \\
\hline Initial Hb & 1.012 & $0.997-1.027$ & 0.111 & & \\
\hline Perioperative Hb & 1.017 & $1.002-1.033$ & 0.027 & & & \\
\hline Initial WBC & 0.953 & $0.849-1.068$ & 0.407 & & & \\
\hline Perioperative WBC & 1.006 & $0.891-1.137$ & 0.922 & & & \\
\hline Initial TB & 0.999 & $0.997-1.001$ & 0.516 & & & \\
\hline Perioperative TB & 1.004 & $1.001-1.007$ & 0.016 & 1.004 & $1.001-1.007$ & 0.017 \\
\hline Initial GGT & 1.000 & $1.000-1.000$ & 0.860 & & & \\
\hline Perioperative GGT & 1.000 & $1.000-1.001$ & 0.893 & & \\
\hline Initial Na & 0.936 & $0.867-1.011$ & 0.091 & & \\
\hline Perioperative Na & 0.990 & $0.916-1.071$ & 0.806 & & \\
\hline Initial PT & 0.962 & $0.841-1.100$ & 0.567 & & \\
\hline Perioperative PT & 0.915 & $0.746-1.123$ & 0.395 & & \\
\hline
\end{tabular}

HR: hazard ratio, Cl: confidence interval, GGT: gamma-glutamyl transferase.

\section{Figures}

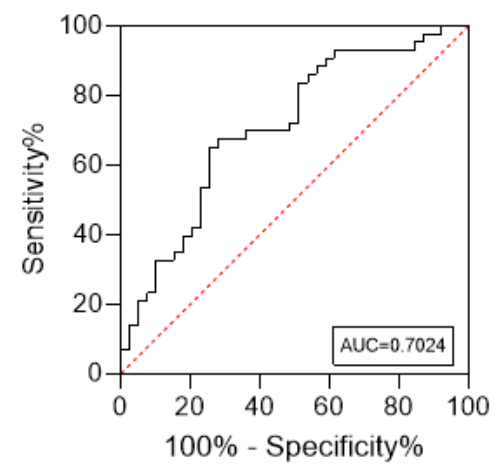

Figure 1

The ROC curve. This analysis shows that the best cutoff of preoperative TB for discriminating patients with complications from those without complications is $162 \mu \mathrm{mol} / \mathrm{L}(P=0.002$, sensitivity $62.8 \%$, specificity $74.4 \%)$, with an area under the ROC curve $(A U C)=0.7024$ [95\% Cl $0.588-0.816]$. 